\title{
EXPERIMENTAL STUDIES ON THE RELATIVE IMPORTANCE OF CONCENTRATION AND DURATION OF EXPOSURE TO DUST INHALATION
}

\author{
BY \\ B. M. WRIGHT \\ From the Pneumoconiosis Research Unit, Llandough Hospital, Penarth, Glamorgan
}

(RECEIVED FOR PUBLICATION JULY 13, 1957)

In a previous paper (Wright, 1953a) reasons have been given for believing that the best measure of the amount of inhaled dust that will be retained in the lungs is the product concentration $\times$ time. This conclusion was based on theoretical considerations of the mechanism of dust inhalation and retention, and on the experience of workers who have studied the correlation between dust exposure and the incidence of pneumoconiosis in men. It has frequently been suggested that the question should be studied experimentally, but no such experiments appear to have been recorded. Although the experimental approach to the question seems at first sight to be an obvious one, there are serious technical difficulties in exposing animals for sufficiently long periods to controlled dust concentrations, and in measuring their exposure. In this paper the way in which these difficulties have been overcome will be described, and an account given of three experiments designed to determine the relative importance of time and concentration in dust exposure.

\section{Technique}

Animal Exposure.-A brief account of the method of exposing animals for prolonged periods to controlled dust concentrations has already been given (King, Wright, Ray, and Harrison, 1950), but considerable technical improvements have been made since then, and the method is now well established. As the technique of experiments of this kind may have a considerable influence on the result it is necessary to describe it in considerable detail.

The basic principle of the method is to keep the animals in a cabinet and pass through it a stream of air to which dust is added at a controlled rate. The animals are normally exposed overnight for 20 hours (2 p.m. to 10 a.m.) for five days a week. This arrangement has the double advantage of using a high proportion of the rather short life-time of most experimental animals, and of making the routine care both of the animals and of the apparatus much easier, since about four hours of each working day are available for the purpose. As the ex- posures are often carried on for periods of a year or more, this routine care is of particular importance. So large a part of the exposure takes place at night when the cabinets are unattended that much care and thought have had to be given to certain technical features of the apparatus which are often neglected; these will now be considered in detail.

Exposure Apparatus.-The complete exposure apparatus comprises the following parts:

(1) Compressed air and vacuum supply

(2) Dust feed mechanisms

(3) Dusting cabinets

(1) Compressed Air Supply.-A clean and dry compressed air supply is absolutely essential, as impurities in the air are one of the commonest causes of difficulty in carrying out dusting experiments. The most common and important impurities are oil (with which most ordinary supplies are contaminated) and excessive water vapour, which may condense in the dust feed mechanism. A pressure of 10 to $15 \mathrm{lb}$./sq.in. is required, and the most satisfactory and economical source has been found to be a compressor of the Rootes type (Lucker, 1950) designed to operate without lubrication of the pumping chamber, so that it delivers completely oil-free air. The compressor is housed in an out-building, as it is rather noisy, together with the vacuum blower used for drawing air out of the cabinets. The general layout is shown in Fig. 1.

Air is drawn in through a filter (1) by the compressor (2) which is connected through a short length of rubber hose (3) (to reduce noise transmission) to a reservoir (5), which in turn leads to the compressed air main (9).

The reservoir smooths the air flow and also contains water-cooling coils (not shown) to remove excess moisture, which can be drained off periodically through the cock (6). The air pressure is adjusted by means of the pressure gauge (7) and blow-off valve (8) according to the amount of air required.

The vacuum blower (11) is of the centrifugal type and gives a vacuum of about 15 inches of water. Like the compressor it is connected by rubber hose (12) to the vacuum main (14) and its outlet (13) should preferably be on the opposite side of the building to the inlet of the 
Fig. 1.-Layout of the blower room.

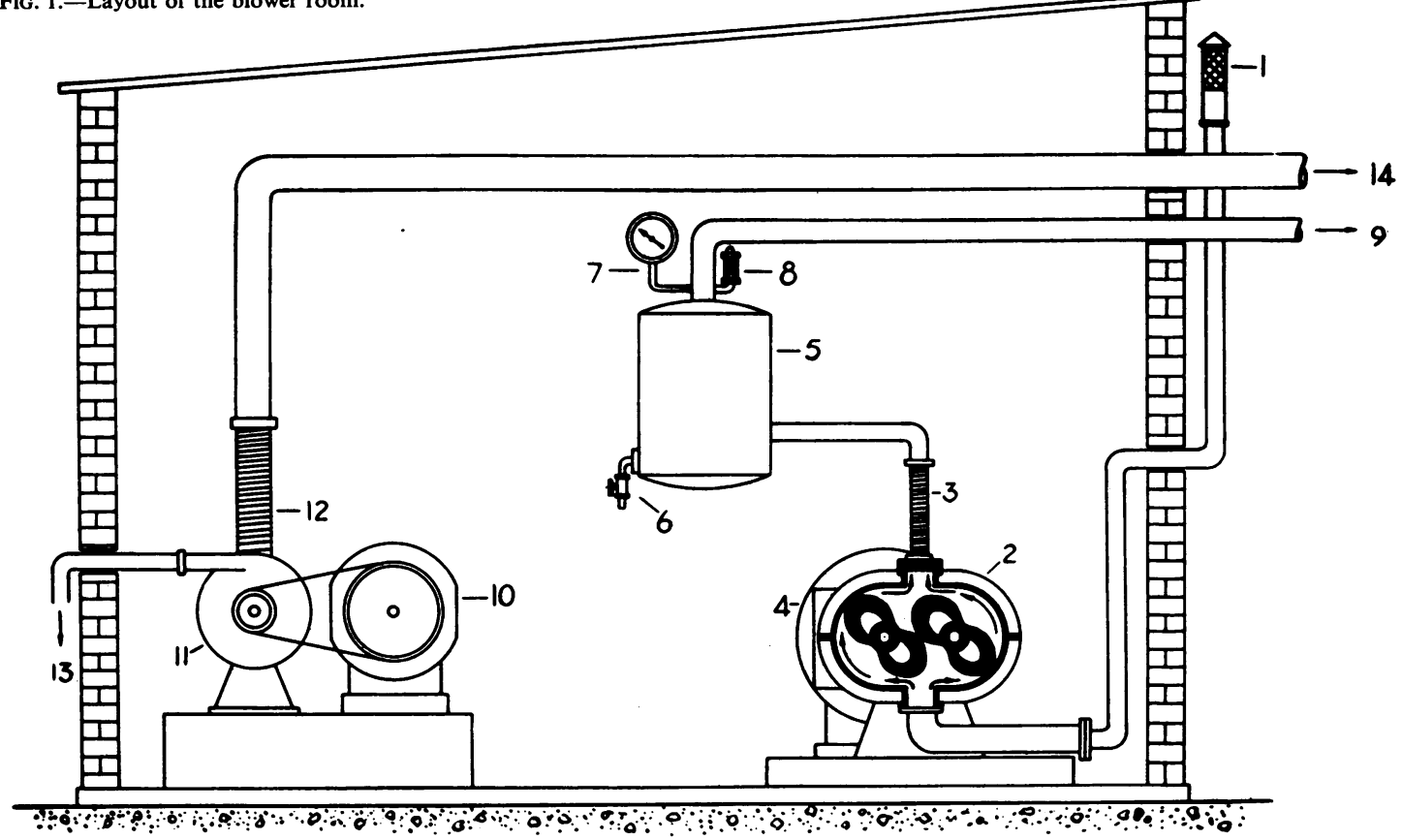

compressor. If suitably chosen and efficiently installed, both machines are capable of running for years without any attention except periodical oiling and greasing.

(2) The Dust Feed Mechanism.This mechanism has been described in detail in a previous publication (Wright, 1950) and its form has remained the same, with only minor improvements and modifications. Its fundamental principle consists of using dust compressed into a cake, and feeding it by a micrometer device against a cutting edge which scrapes a very thin layer off into the air stream. The dust concentration can be varied by adjusting the rate of feed, and any desired level reached by trial and error. Once the appropriate feed rate has been found, the dust concentration can be kept at the same average level indefinitely, so long as the dust used remains of the same size distribution. The present form of the instrument is shown in Fig. 2.

(3) The Dusting Cabinets.-These are similar to those previously described, the main modification being that the pyramidal top has been truncated. The general

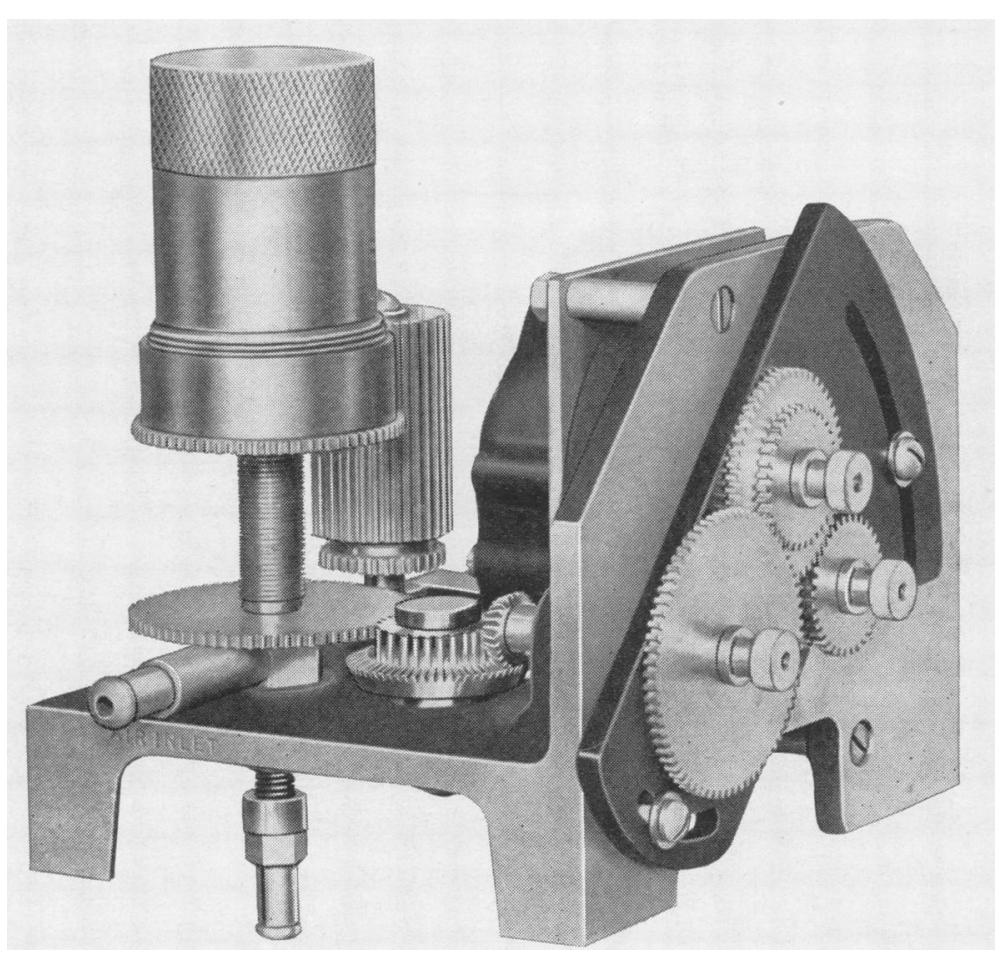

Fig. 2.-The Wright dust feed mechanism. 


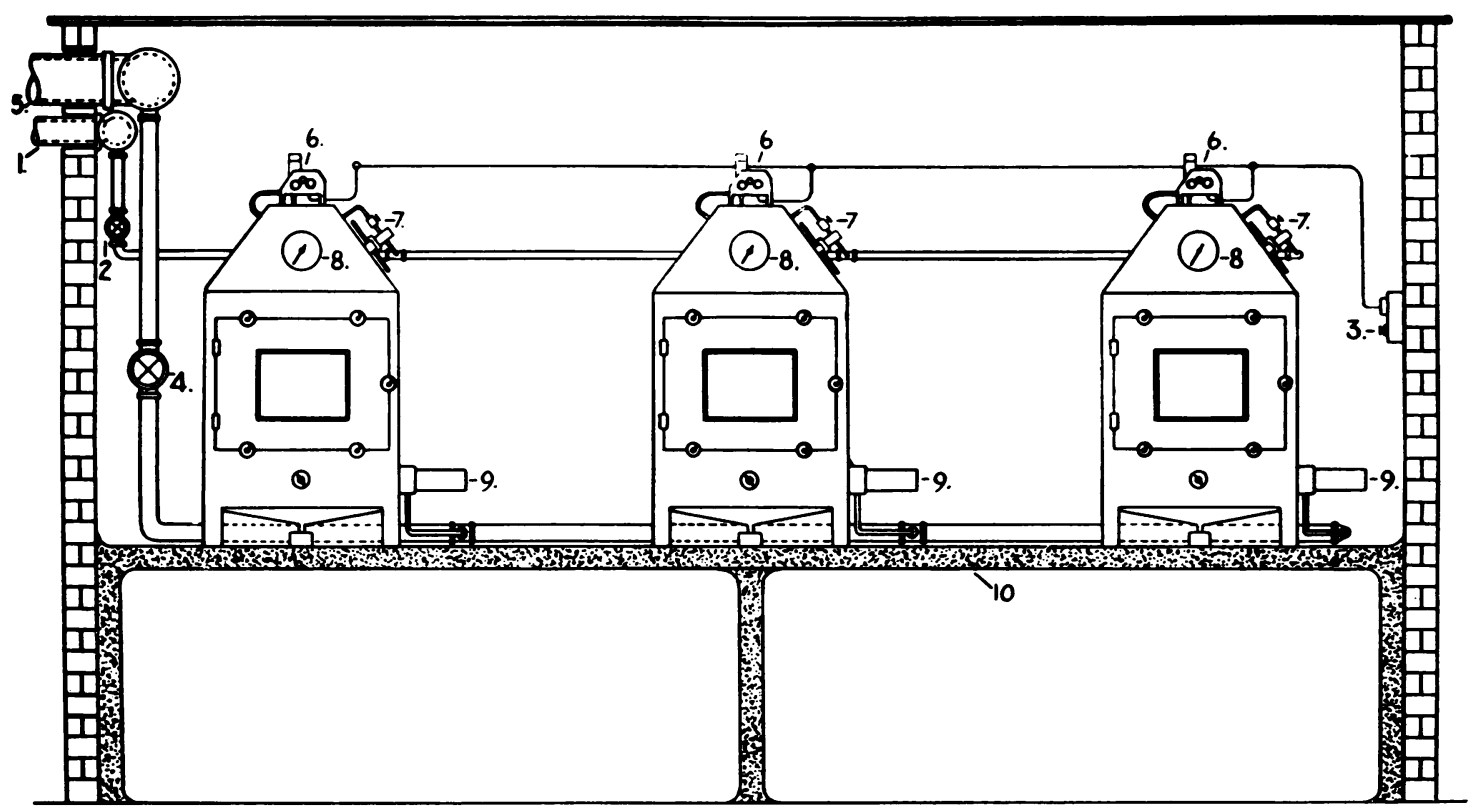

FIG. 3.-General arrangement of the dusting cabinets.

arrangement of the cabinets is shown in Fig. 3. The cabinets (Fig. 4) are made of sheet metal with glass or perspex windows. The door is sealed with sponge rubber, and secured by means of " burglar proof" type window catches (not shown). The cabinet is not completely airtight, so arrangements are made to ensure that the pressure within it is always very slightly below atmospheric, to minimize the leakage rate and prevent dust escaping into the room. This is done in the following way: air is drawn from the cabinet through the orifice (15) at the bottom and through the Soxhlet thimble filter (16) to the vacuum main (19). The rate of flow through the filter is considerably greater than the rate of flow into the cabinet, but the excess air is drawn in through three large bypass holes (18) so that the suction head that is applied at the orifice (15) is negligibly small. The system works well, so long as the dust concentrations used are not so high that the filter clogs and reduces the output rate below the input rate in less than 20 hours.

Because of the danger of the animals asphyxiating if the air supply fails (e.g., during a power cut) each cabinet is fitted with a spring-loaded safety hatch (4) which is normally held closed by the compressed air pressure, but opens if it fails.

The air flow into the cabinet through the dust feed mechanism (9) is controlled by the valve (5) and flow meter (6) (made from an air speed indicator).

The rate of air flow was originally fixed by reference to the ventilation rate of the animals. According to Guyton (1947) the minute volume of an average rat is about $100 \mathrm{ml}$., so a group of 20 rats would breathe 2 litres/minute. In order to keep the carbon dioxide level in the cabinets at a reasonable level, a volume con- siderably greater than this is required, and a figure of 10 litres/minute was chosen. This keeps the carbon dioxide level at about $1 \%$.

The animals (usually rats) are kept in open cages 20 in. $\times 20$ in. $\times 9$ in. which will accommodate up to 20 young rats. The cages have mesh bottoms and rest on runners, with a removable tray for droppings below. Food and water are provided in excess, and " cannibalization" of dead animals is thus kept to a low level, though unfortunately not entirely eliminated. The general health of the animals is good; the exposed ones often live longer on the average than their unexposed fellows, when in moderate concentrations of inert materials such as coal dust, possibly because of the uniform air-conditioned environment in which they live.

Measurement of Dust Exposure.-In experiments of mainly pathological interest, such as that described by King et al. (1950) it has been found sufficient to take some preliminary measurements with a thermal precipitator and adjust the mechanism until the desired concentration level is obtained. It can be assumed that this concentration has been maintained at about the right level all night, so long as the air flow is still within $10 \%$ of the proper level, and the dust feed mechanism is still running freely next morning. A snap check is usually made an hour after starting, and before stopping exposure in the morning, with a simple filter paper stain device. This has no pretensions to accuracy but guards against human errors such as forgetting to switch on a mechanism.

For the purpose of the particular experiments to be described, a more reliable and accurate method was required, and therefore a long-term continuous sampler 


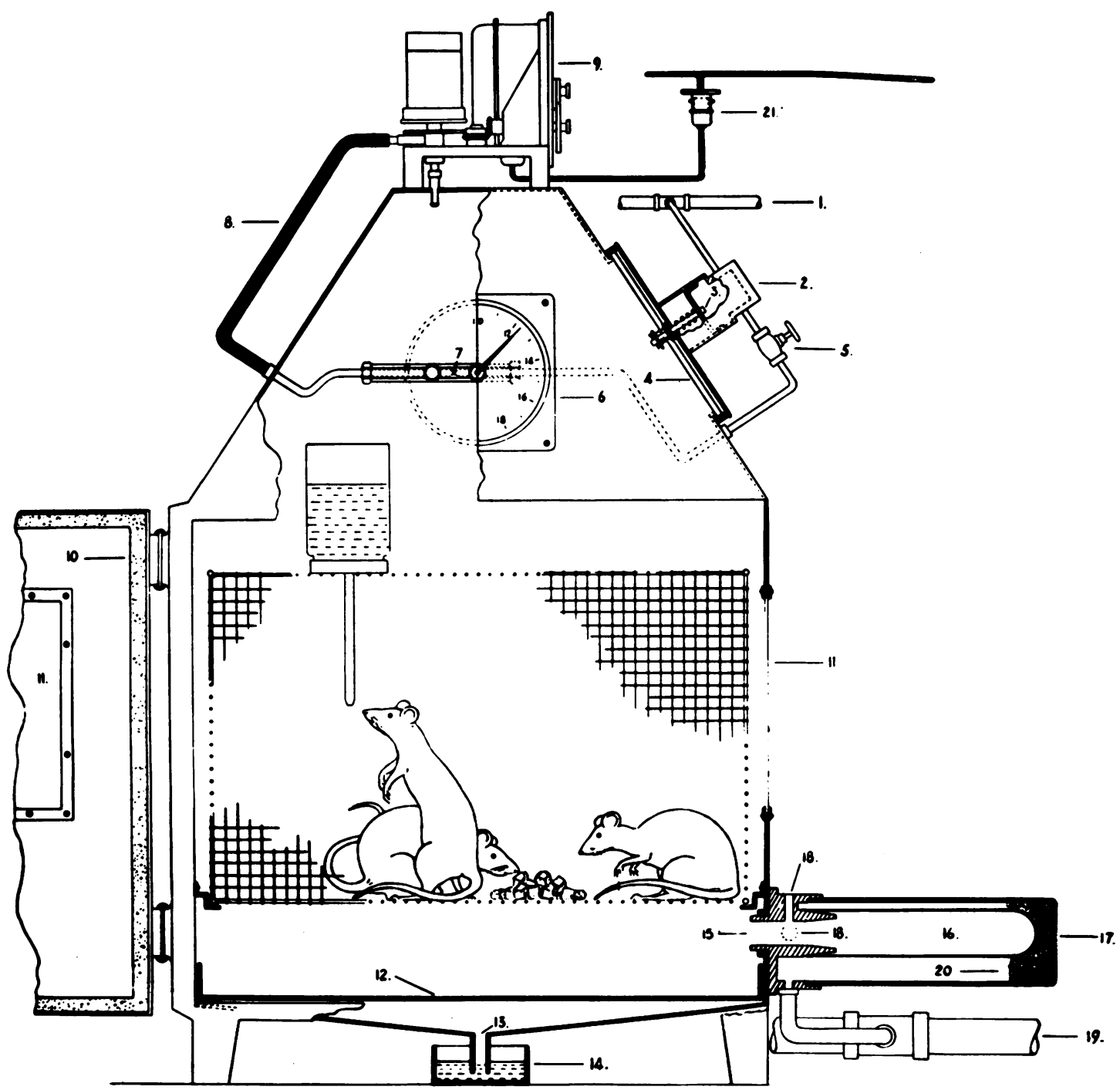

Fig. 4.-Detail of a dusting cabinet.

was developed. This consisted of a gravimetric thermal precipitator (Wright, 1953b) through which air was drawn from the cabinet at $100 \mathrm{ml}$./minute (approximately the minute volume of a rat), and which deposited the dust on an aluminium plate on which quantities of the order of $7 \mathrm{mg}$. could be easily weighed with sufficient accuracy.

Gravimetric sampling is not a satisfactory method of measuring dust exposure unless precautions are taken to exclude from the sample particles and aggregates too large to be retained in the lungs. Such particles and aggregates may be present to a variable extent, and may constitute more than $50 \%$ by weight of the airborne dust, even when the cloud is produced from material which is all nominally within the "respirable" size range (see
Wright, 1953a). Some form of size selector must therefore be fitted to the intake of the sampler to prevent particles outside the respirable range from reaching the collecting plate. In the first experiment to be described the sampler was fitted with a rather crude and unsatisfactory cylindrical elutriator, but in later experiments a simple horizontal duct of the type specified by Hamilton and Walton (1952) was used. This was made by flattening a piece of thin-walled brass tubing to give a duct $1 \mathrm{~mm}$. high, $1.5 \mathrm{~cm}$. wide, and $7 \cdot 3 \mathrm{~cm}$. long. The floor area of $10.96 \mathrm{~cm}^{2}$ gives a theoretical cut-off of $50 \%$ of $5 \mu$ diameter spheres of unit density at a flow rate of $100 \mathrm{ml}$./ minute. Tests with a glass sphere cloud (Wright, 1954) showed good agreement with the theory. The reasons for 


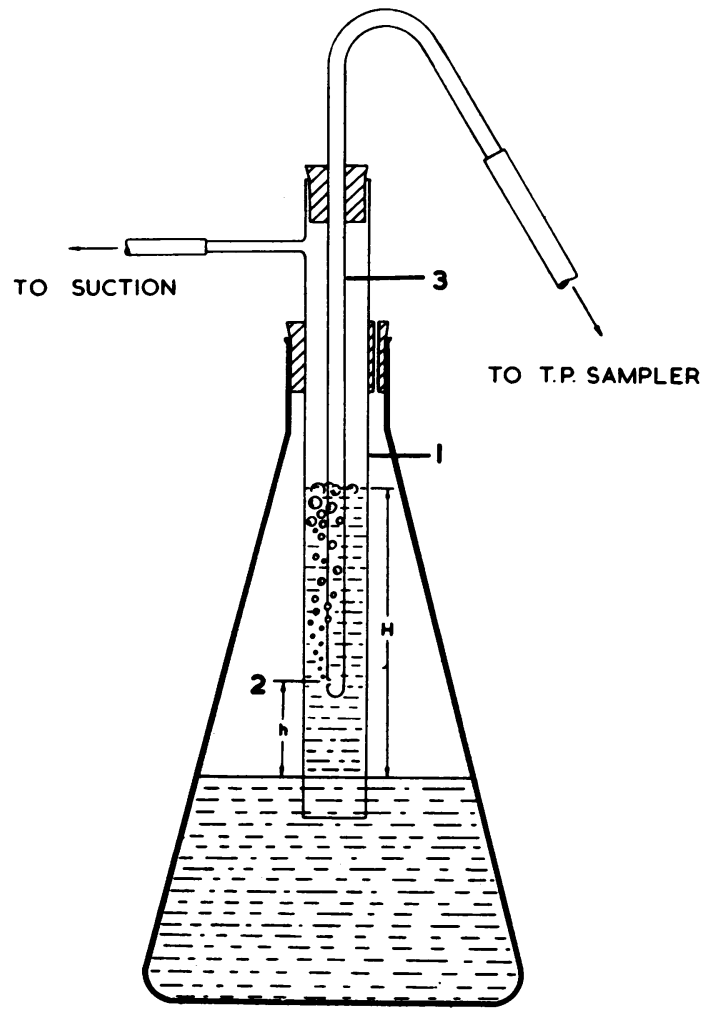

FIG. 5.-Constant-level suction device.

choosing this cut-off and the question of its suitability will be discussed later.

This simple elutriator was found to work satisfactorily, except for a tendency to clog at the mouth, where the large particles and aggregates accumulated. The mouth was, therefore, belled in a vertical plane, which is permissible since the height of the duct is immaterial (Hamilton and Walton, 1952) and this overcame the difficulty.

The control of the flow through the sampler was fairly critical, since not only was the aspirated volume calculated on the assumption of a constant flow, but also variations in the flow rate would affect the cut-off, and consequently the mass concentration of the dust penetrating to the thermal precipitator. Suction was therefore taken from the vacuum main, which was subject only to small long-period variations, and stabilized by means of the hydrostatic constant head device shown in Fig. 5. Provided that the diameter of the inner tube (1) is small compared with that of the flask and that the loss of water by evaporation is negligible, variations in the available suction head $(\mathrm{H})$ will not affect the effective suction head $(h)$, because the head of water above the orifice (2) exactly cancels the difference between $H$ and $h$. Of course, $H$ must never at any time fall below $h$, but this is easy to arrange. The effective suction head $h$ can be adjusted by sliding the inlet tube (3) up and down in the cork. With a head of about $20 \mathrm{~cm}$. water, a flow of $100 \mathrm{ml}$./minute is given by an orifice about $\mathbf{0 . 2 5} \mathrm{mm}$. in diameter. Such a small orifice would normally be liable to clog, but since the air passing through it has all passed through the thermal precipitator it is completely free of dust, and the flow can be maintained constant within $5 \%$ for 20 hours quite easily. The flow was adjusted and checked with a rotameter at the beginning of each exposure, and again at the end. The rotameter itself was made in the laboratory and calibrated with a bubble flowmeter.

\section{Plan of Experiments}

The general plan of all the experiments was the same; two groups of rats were exposed in the same cabinet, one (A) for the usual 20-hour period overnight, and the other (B) for two hours in the morning. Before starting the actual exposure experiment, the dust feed rate was adjusted so that the dose administered, as measured with the sampler, was the same in both cases; i.e., the dust concentration in the short period B was 10 times that in the long period $\mathrm{A}$.

The two doses were measured daily, and the total doses summed up from day to day. If the difference between the total doses exceeded one day's dose, either one mechanism was adjusted or an occasional exposure was missed, so as to keep the totals as close as possible. The daily dose averaged about $7 \mathrm{mg}$., varying from 4 to $10 \mathrm{mg}$. on occasions.

At the end of the experiment all surviving animals were killed at the same time, within a few days of the end of exposure, and the dust was extracted from their lungs and weighed. It was expected that there would be some variation in the amount of dust in the lungs in different animals within the same group, which would be likely to be to some extent correlated with their body weights, so all the animals were weighed at the beginning of the experiment, identified by ear-punching, and weighed again at the end. As will be seen, this precaution was well worth while as the correction for body weight, using covariance analysis (Fisher, 1934), considerably reduced the scatter.

\section{Results}

Experiment I.-Two groups of 14 young male rats were exposed for five days a week over a period of three months. One animal in Group A was accidentally killed, but otherwise both groups survived to the end of the experiment. Ten animals in each group were used for dust extraction, the lungs of the remainder being examined histologically. The dust used was "levigated alumina", a form of synthetic alumina $\left(\mathrm{Al}_{2} \mathrm{O}_{3}\right)$ made for fine grinding and polishing. This was chosen as being readily available and known to be of very low solubility and pathologically inert, and at the same time easily estimated in the lungs by simple ashing. Although this method of estimating the lung dust was used initially, it was eventually decided to estimate the $\mathrm{Al}_{2} \mathrm{O}_{3}$ content of the ash chemically, so as to eliminate possible 
TABLE 1

RESULTS IN EXPERIMENT I

\begin{tabular}{|c|c|c|c|c|c|}
\hline Group A (503 mg. at & low concentration & exposure) & Group B $(503.6 \mathrm{mg}$. at & igh concentra & ort exposure) \\
\hline \multirow{2}{*}{ Body Weight } & \multicolumn{2}{|c|}{$\mathrm{Al}_{2} \mathrm{O}_{3}$ (mg.) } & \multirow{2}{*}{$\begin{array}{l}\text { Body Weight } \\
\text { (g.) }\end{array}$} & \multicolumn{2}{|c|}{$\mathrm{Al}_{3} \mathrm{O}_{3}$ (mg.) } \\
\hline & Uncorrected & Corrected & & Uncorrected & Corrected \\
\hline $\begin{array}{l}235 \\
245 \\
260 \\
295 \\
300 \\
254 \\
255 \\
270 \\
235 \\
260\end{array}$ & $\begin{array}{l}\mathbf{6 7 \cdot 2} \\
69 \cdot 3 \\
74 \cdot 5 \\
76 \cdot 2 \\
78 \cdot 3 \\
80 \cdot 2 \\
63 \cdot 0 \\
70 \cdot 0 \\
53 \cdot 9 \\
78 \cdot 2\end{array}$ & $\begin{array}{l}\mathbf{7 9 \cdot 0} \\
\mathbf{7 7 \cdot 7} \\
\mathbf{7 7 \cdot 7} \\
\mathbf{6 7 \cdot 5} \\
\mathbf{6 7 \cdot 9} \\
85 \cdot 5 \\
\mathbf{6 7 \cdot 5} \\
\mathbf{6 9 \cdot 8} \\
\mathbf{6 5 \cdot 7} \\
81 \cdot 4\end{array}$ & $\begin{array}{l}255 \\
255 \\
285 \\
290 \\
280 \\
280 \\
250 \\
325 \\
305 \\
255\end{array}$ & $\begin{array}{l}58 \cdot 3 \\
49 \cdot 1 \\
55 \cdot 0 \\
59 \cdot 7 \\
59 \cdot 2 \\
65 \cdot 4 \\
46 \cdot 7 \\
84 \cdot 1 \\
68 \cdot 3 \\
41 \cdot 7\end{array}$ & $\begin{array}{l}63.2 \\
54.0 \\
49.7 \\
52.7 \\
55.6 \\
61.8 \\
53.4 \\
65.1 \\
56.1 \\
46.6\end{array}$ \\
\hline Mean & $71 \cdot 1$ & $74 \cdot 0$ & & $58 \cdot 7$ & 55.8 \\
\hline$\overline{\text { S.D. }}$ & $8 \cdot 2$ & $7 \cdot 1$ & & $12 \cdot 1$ & $5 \cdot 9$ \\
\hline Coefficient of variation & $11 \cdot 5 \%$ & $9 \cdot 6 \%$ & & $20 \cdot 6 \%$ & $10 \cdot 6 \%$ \\
\hline Percentage retention & & $14 \cdot 7$ & & & 11.1 \\
\hline
\end{tabular}

errors caused by the need to make an allowance for the " intrinsic" lung ash.

Although the animals were weighed both at the beginning and end of the experiment, it was found that either weight could be used for " correction", so the dead weight was chosen because it was rather more easy to determine accurately.

The dose of dust administered to both groups, as measured by the sampler and the amounts found in the lungs of the rats (estimated chemically as $\mathrm{Al}_{2} \mathrm{O}_{3}$ ), are given in Table 1.

The following points may be noted at this stage:-

(1) The effect of correcting for body weight is to reduce the scatter of the results from $11.5 \%$ to $9.6 \%$ in Group A, and from $20.6 \%$ to $10.6 \%$ in Group B.

(2) The animals in Group A retained $14.7 \%$, and in Group B $11.1 \%$ of the dust collected by the sampler.

(3) The ratio between the average amount of dust retained by the animals in Group $A$ and those in Group B is $1 \cdot 325(95 \%$ confidence limits $1 \cdot 20-1 \cdot 47)$.

Experiment II.-This was a repetition of Experiment $I$, except that, as mentioned earlier, a different type of size selector was fitted to the sampler and also a different technique was used for extracting the dust from the lungs. It was found that $\mathrm{Al}_{2} \mathrm{O}_{3}$ could be easily and quantitatively extracted from the lungs by a technique which had been used for extracting coal dust. This technique, which has been developed by Mr. T. J. H. Cooke, is given in the Appendix.

During the experiment the original supply of " levigated alumina" ran out, and the new supply, obtained from the same makers, was found to be considerably coarser. For this reason it was necessary to run the experiment for two months longer, and the dose of $500 \mathrm{mg}$. obtained in the first experiment could not be reached. Two groups of 10 animals were used, and they all survived to the end of the experiment.

The results are given in Table 2.

TABLE 2

RESULTS IN EXPERIMENT II

\begin{tabular}{|c|c|c|}
\hline & Group A & Group B \\
\hline $\begin{array}{l}\text { Exposure } \\
\text { Dose } \\
\text { Weight of dust in lungs } \\
\text { (standardized for body } \\
\text { weight) } \\
\text { Percentage retention }\end{array}$ & $\begin{array}{l}\text { Low concentration } \\
427 \mathrm{mg} \text {. } \\
32 \mathrm{mg} \text {. } \\
\text { (S.D. } 5.8 \mathrm{mg} \text {.) } \\
7.5\end{array}$ & $\begin{array}{l}\text { High concentration } \\
440 \mathrm{mg} \text {. } \\
23 \mathrm{mg} \text {. } \\
\text { (S.D. } 2 \cdot 7 \mathrm{mg} \text {.) } \\
5.2\end{array}$ \\
\hline
\end{tabular}

Owing to a technical error the exposure of the two groups was slightly different so that the comparison between them can only be made on a percentage retention basis. On this basis the ratio A/B is 1.37 (confidence limits $1 \cdot 13-1 \cdot 66$ ). This ratio is very close to that found in the first experiment $(1 \cdot 325)$. On the other hand, the percentage retention is only about half that observed in the first experiment. This difference may have been partly due to the change in the size distribution of the dust, and partly to the change of size selectors. A comparison between the new and old size selectors showed that the new one cut off about $10 \%$ more, by weight, than the old. Owing to the unsatisfactory nature of the old size selector this anomaly was not further investigated, but the question of size selection and the effect of size distribution will be considered again later.

Experiment III.-The first two experiments were open to criticism on the ground that the concentrations used, even in the " low " concentration cabinet, were considerably higher than those commonly 
found in human exposure, being about $60 \mathrm{mg}$. per cubic metre in the A experiment, and 600 in the B. Comparable figures, using a basically similar size selecting sampler in a moderately dusty coal-mine, would be $20 \mathrm{mg}$./cu.m. (Wright, 1954). A third experiment was therefore carried out in which the two groups were exposed to much lower concentrations, the dose used in the previous experiments being administered in a week instead of a day, and the experiment being carried out for 18 months instead of three and five months, thus reducing the dust concentrations by a factor of about 5 .

It was also decided to use coal-dust instead of levigated alumina for a number of reasons:-

(1) The original reason for using alumina-its ease of estimation chemically or by ashing - was no longer important since a dust extraction method was being used.

(2) Our accumulated experience with a variety of coal dusts and other purer forms of carbon suggested that coal-dust was pathologically as inert as alumina and was therefore equally suitable for use in longterm experiments where any kind of specific pathological response was undesirable as it would confuse the issue.

(3) A device, the " turbine grinder ", for producing large quantities of fine coal-dust of constant size distribution had already been developed (Wright, 1953c) and so a steady supply of dust for the whole experiment could be assured.

For these reasons " turbine ground" steam coal was used and the concentration was adjusted so that the dose given in each experiment was about $7 \mathrm{mg}$. in five days. The gravimetric thermal precipitator with a horizontal elutriator was used exactly as in Experiment II, except that the samples were weighed only once a week instead of daily.

The experiment was started on July 26,1954 , and ended on December 30, 1955. As was to be expected in an experiment of this long duration there were occasional breakdowns, and some casualties among the animals. In particular, five of Group A died early in the experiment from an infection, and three of Group B died near the end of the exposure. It was noticeable that the Group B animals were less healthy than those in Group A; most of them lost weight towards the end, and at necropsy much more bronchiectasis was found among them than in Group A. Apart from this, no difference in the histological appearances between the two groups was noted in this or in either of the two previous experiments. Although it is tempting to attribute this difference in health to the difference in dust exposure, there is in fact another explanation for which there is supporting evidence. The Group B animals only spent two hours a day in the cabinet, while the Group A were in it for most of their lives. The latter were therefore in the uniform sheltered environment and conditioned air which, as already noted, seems to have a beneficial effect on the health of the animals, while the former were not, and this could completely account for the difference.

A number of mechanical breakdowns also occurred, but by suitable adjustments of the dust feed rate it was possible in the end almost to equalize the doses of the two groups.

Eighteen young male rats were used in each group, chosen by random numbers from a single group of 36. Of these 11 survived to the end of the experiment in Group A, and 12 in Group B. All the animals were earmarked and weighed at the beginning of the experiment and weighed again at three-monthly intervals, and at death. Two animals in each group were used for histological study; the results for the remainder are given in Table 3.

TABLE 3

RESULTS IN EXPERIMENT III

\begin{tabular}{|c|c|c|}
\hline & Group A & Group B \\
\hline $\begin{array}{l}\text { Exposure } \\
\text { Dose } \\
\text { Weight of dust in lungs } \\
\text { (standardized for body } \\
\text { weight) } \\
\text { Percentage retention }\end{array}$ & $\begin{array}{l}\text { Low concentration } \\
444 \mathrm{mg} \text {. } \\
97.4 \mathrm{mg} . \\
\text { (S.D. } 15.2 \mathrm{mg} \text {.) }\end{array}$ & $\begin{array}{l}\text { High concentration } \\
446 \mathrm{mg} . \\
82.5 \mathrm{mg} . \\
\text { (S.D. } 12.9 \mathrm{mg} \text {.) }\end{array}$ \\
\hline
\end{tabular}

The body weight used for standardization was the average of the three-monthly weights up to and including the maximum weight reached.

The ratio of the percentage retentions is 1.18 (95\% confidence limits 1.07-1.31). Although this ratio is rather smaller than those found in the two previous experiments it cannot be said that it is significantly different.

\section{Discussion}

The immediate conclusion that can be drawn from these experiments is that, under the described conditions, a very large difference in the concentration and duration of exposure produced a relatively small difference in the amount of dust accumulating in the lungs. The further conclusion, that a high-concentration, short-duration exposure produced from $20 \%$ to $30 \%$ less effect, is somewhat more doubtful as its validity depends on the assumption that the sampling instrument was giving a true measure of the amount of dust that would be retained in the lungs. This in turn depends on the assumption that its size selector had filtration properties similar to those of the upper respiratory tract of rats, so that the dust penetrating to the thermal precipitator would be similar in size distribution to that which 
would be deposited in the alveoli. Although a certain amount of information is available about the filtration properties of the upper respiratory tract in both men (Davies, 1949; Wilson and La Mer, 1948) and animals (Shoshkes, Banfield, and Rosenbaum, 1950; Palm, McNerney, and Hatch, 1956) none of this information enables the size-selecting characteristics of the respiratory tract to be specified with any great accuracy. From a practical point of view, in fact, as much useful information can be derived by studying the dust obtained from the animals' lungs and noting that, like the dust extracted from human lungs, it contains very few particles indeed over about $7 \mu$ average diameter. It was for this reason that it was decided to use the standard elutriator specified by Hamilton and Walton. This has no pretensions to simulate, except in the most general way, the size-selecting characteristics of the upper respiratory tract, but it has the great merit of being easily specified theoretically, and reproduced mechanically. At the same time it separates particles by the same physical mechanism as operates in the respiratory tract, and gives a cut-off at about the same level.

Unfortunately it can be shown theoretically, and has been found experimentally, that the weight of dust collected by a sampler fitted with a size selector will vary considerably according to the cut-off level chosen, and also that the amount of this variation will differ with clouds of different size distribution.

If, therefore, the size selector used was in fact very different in its properties from the upper respiratory tract of the rats, the difference in the amount of dust retained by the two groups of animals might be accounted for, wholly or partly, by a difference in the size distribution of the high and low concentration clouds. Such a difference might well be present, due to more aggregation occurring in the high concentration cloud.

Experimental.-To investigate this question some sampling studies were made to see the effect of using size selectors with different characteristics in the two types of experimental cloud. The sampling was carried out exactly as in the main experiment, and a group of animals was left in the cabinet so that any effects of heat and humidity on the size distribution of the cloud would be reproduced.

Two gravimetric thermal precipitators were used, one fitted with the standard size selector having a cut-off of $50 \%$ at $5 \mu$ diameter, and the other with a longer elutriator of the same pattern having a cut-off of $50 \%$ at $3 \mu$ diameter.

Sampling was first carried out in the aluminium oxide clouds. As was to be expected, the dose measured with the $3 \mu$ selector was smaller than that measured with the $5 \mu$ selector, but in addition the difference was greater in the $B$ cloud than in the A. Had, therefore, a $3 \mu$ elutriator been used instead of a $5 \mu$, the dose given to the two groups would have been found to be different. Expressed as a ratio $\mathrm{A} / \mathrm{B}$ this difference was $1 \cdot 20$, which might therefore account partly for the difference in the percentage retention of the two groups.

Similar studies were then made in the coal-dust clouds taking weekly instead of daily samples. The results were similar, but the difference between the two clouds was smaller, the ratio being only $1 \cdot 03$. This again could account for a small part of the difference in retention of the two groups of animals.

In both cases only 15 sets of samples were taken and there was sufficient scatter to prevent the observed difference being significant at the $5 \%$ level; as both studies showed a difference in the same direction, which was also that expected, it is probable that a sufficiently prolonged trial would establish a significant difference eventually, but in view of the small magnitude of the difference such a trial seemed hardly worth while, especially in the coal-dust clouds, where only one sample a week could be obtained. For the same reason direct studies of the size distributions by microscopy were not carried out because it was felt that the results might well be ambiguous and not worth the trouble involved.

It seems likely that the use of a size selector with an even lower cut-off than $50 \%$ at $3 \mu$ might further increase the difference between the dose measured in the two clouds, but it is hard to see how the use of such a size selector could be justified on the available evidence. Probably the best data are those of Shoshkes et al. (1950) who found that the mass median diameter of corn oil droplets (density 0.9 ) penetrating to the alveoli of mice was $2 \cdot 9 \mu$, the maximum observed diameter being $6 \mu$. Some experiments of my own, using glass spheres (density $2 \cdot 25$ ), gave very similar results, the cut-off, for a variety of animals, including mice, rats, cats, rabbits, and a small monkey, being at about $4 \mu$, which is equivalent to $6 \mu$ at unit density.

A horizontal elutriator with a cut-off of $50 \%$ at $3 \mu$ has a cut-off of $100 \%$ at $4 \cdot 25 \mu$, which on this evidence at least is already a good deal too low, and the standard elutriator with a cut-off at $7.07 \mu$ is nearer the mark.

It may be concluded therefore that at best only a small part of the difference in dust retention by the two groups could possibly be accounted for by the use of an inappropriate size selector on the sampler, and that most of the difference must be considered real.

It is possible to speculate at length on the physiological mechanisms which might account for the difference in retention between the two groups, but since this difference is small compared with the difference in the type of exposure it would be profitless to do so. One possible explanation that might 
be advanced is that the high incidence of bronchiectasis in the B group might have led to the loss of dust in the exudate. Since, however, bronchiectasis of any degree was only observed in the long-term experiment, in which the difference in retention between the two groups was least, this seems an unlikely explanation.

It is tempting to attribute the marked increase in the percentage retention in Experiment III, as compared with that in I and II, to the more prolonged exposure to lower concentrations, but it may equally well have been due to a difference in the size distribution of particles in the dust clouds.

A further point of interest is that the reduction in the dose that would be produced by the use of a $3 \mu$ elutriator would only double the percentage retention, so that no conceivable elutriator could bring it up to $100 \%$. This is, of course, to be expected on two grounds. First, all the available evidence suggests that the alveolar retention of the most penetrating particle size, about $1 \mu$, is only $50 \%$; and second, an unknown proportion of particles which penetrate to the alveoli may be subsequently removed by phagocytes and transported to the ciliated epithelium and so out of the lung.

It is obvious, however, that much more needs to be done to establish the pattern of the relationship between dust exposure and retention. The main object of this paper has been to show how such investigations can be carried out, and to stimulate others working in the field to explore this part of it.

\section{Summary}

Details are given of an experimental technique for exposing groups of animals for long periods to the inhalation of dust clouds of controlled concentration, and of a method of measuring their average exposure.

Three experiments are described in which two groups of rats were exposed to the inhalation of dust, one group being exposed for 20 hours a day and the other for two hours to a concentration 10 times greater, so that the two groups both received the same daily dose.

In the first two experiments the animals were exposed to relatively high concentrations of aluminium oxide dust over a period of a few months, and in the third to moderate concentrations of coal dust for 18 months.

In all three experiments the amount of dust retained by the two groups was very similar, but the high concentration group always retained rather less (20-30\% less) than the low concentration group.

The validity and implications of these findings are discussed.
The techniques and experiments described have been evolved and carried out over a period of about 10 years and it is impossible to acknowledge the assistance of all those who have taken part in the work.

I should like, however, especially to mention my first animal technician, the late Mr. A. S. Appleby, who helped to work out the routine of exposure and whose painstaking care made these prolonged experiments possible.

Mr. N. E. Bevan also has assisted me from the beginning in the development of dust inhalation and sampling techniques, and Mr. M. Williams had charge of the animals in the last experiment.

Mr. T. J. H. Cooke has carried out all the lung dust estimations, except for the $\mathrm{Al}_{2} \mathrm{O}_{3}$ estimations in the first experiment, which were done by Dr. T. G. Morris.

The drawings were prepared by Mr. W. H. Roberts.

I am indebted to Mr. P. D. Oldham for the statistical treatment of the results and for much valuable advice, and to Mr. S. A. Roach for much helpful criticism.

Finally, I am grateful to the former and present Directors of this Unit, Dr. C. M. Fletcher and Dr. J. C. Gilson, for providing the facilities and encouragement to carry out this work.

\section{REFERENCES}

Davies, C. N. (1949). British Journal of Industrial Medicine, 6, 245 Fisher, R. A. (1934). Statistical Methods for Research Workers, 5th ed., p. 280. Oliver and Boyd, Edinburgh.

Hamilton, R. J., and Walton, W. H. (1952). The Selective Sampling of Airborne Dusts. National Coal Board, Scientific Departof Airborne Dusts. National Coal Board, Scientific

King, E. J., Wright, B. M., Ray, S. C., and Harrison, C. V. (1950). British Journal of Industrial Medicine, 7, 27.

British Journal of Industrial Medicine, 7, 27.
Lucker, F. L. (1950). In Chemical Engineers' Handbook, 3rd ed., Lucker, F. L. (1950). In Chemical Engineers' Handbook,
p. 1452, ed. J. H. Perry. McGraw-Hill, New York.

p. 1452, ed. J. H. Perry. McGraw-Hill, New York.
Palm, P. E., McNerney, J. M., and Hatch, T. (1956). A.M.A. Arch. industr. Hith, 13, 355 .

Shoshkes, M., Banfield, W. G., and Rosenbaum, S. J. (1950). Arch. industr. Hyg., 1, 20.

Wilson, I. B., and La Mer, V. K. (1948). J. industr. Hyg., 30, 265.

Wright, B. M. (1950). J. sci. Instrum., 27, 12.

(1953a). British Journal of Industrial Medicine, 10, 235.

(1953b). Science, 118, 195.

(1953c). Chem. and Industry, p. 8.

(1954). British Journal of Industrial Medicine, 11, 284.

\section{A P P E N D I X}

\section{The Extraction of Dust from Tissues}

Principle.-The tissues are dehydrated with alcohol, defatted with ether, and dissolved in hot concentrated $\mathrm{HCl}$. Acid-insoluble material is then removed by centrifugation, dried, and weighed.

Technique.-The tissues are fixed by immersion in absolute alcohol overnight. Any dissection or examination required, e.g., separation of lungs and hilar glands from the heart, is then carried out, and the tissues cut into slices about $2 \mathrm{~mm}$. thick. These are placed in centrifuge tubes and covered with absolute alcohol, and again left overnight. The following morning the alcohol is poured off and replaced by ether, which is changed once and then left overnight. Next morning the ether is poured off, and the tubes filled with hot $\left(50^{\circ} \mathrm{C}\right)$ concentrated $\mathrm{HCl}$. As soon as the residue of the ether has evaporated, the tubes are firmly stoppered and placed in a rack in an 
oven or water-bath at $60^{\circ} \mathrm{C}$ for two to three hours. The rack is shaken from time to time, and the process of solution observed. As soon as all visible aggregates have disappeared, the tubes are removed from the rack and centrifuged at 3000 r.p.m. for two hours. The supernatant should then be clear, but as it is a dark purple or brown colour it must be examined against a very bright light. If it is opalescent, or contains visible suspended matter, the tube should be shaken up again, and the contents divided between two tubes and reheated with more acid. The deposit is washed once in alcohol and then resuspended in a small volume of alcohol, any dust clinging to the side of the tube being carefully washed down. It can then be transferred to a suitable receptacle, dried and weighed. It has been found convenient to use
" bijou" screw-capped bottles, in which the samples can be kept for future reference. Drying is accelerated if these are centrifuged, and most of the supernatant removed.

Notes.-Formalinized tissues can be used, but take longer to dissolve, as do tissues which have been fixed in alcohol for some time.

Defatting is necessary because the fat does not dissolve in the acid but forms a pellicle on centrifuging, in which a good deal of dust is trapped.

The whole process could probably be accelerated by shortening the times of immersion in the second change of alcohol and in ether, but the times given are those actually used in the experiments described. 